\title{
TOMOGRAPHIC PARTICLE IMAGE VELOCIMETRY (TOMOGRAPHIC-PIV): É POSSÍVEL MEDIR CONCENTRAÇÃO DE PARTÍCULAS?
}

\author{
R. L. AMARAL*, G. J. CASTILHO, S.S.V. VIANNA e M. MORI \\ Universidade Estadual de Campinas, Faculdade de Engenharia Química \\ *e-mail: rlamaral @feq.unicamp.br
}

\begin{abstract}
RESUMO
A técnica de Velocimetria por Imagem de Partícula (PIV) é uma técnica de medida não intrusiva utilizada nos último 20 anos para determinar campos de velocidade de escoamentos multifásicos. A Tomographic-PIV determina a distribuição da velocidade em 3D do fluido possibilitando a investigação de estruturas de escoamentos. $\mathrm{O}$ conhecimento da distribuíção de particulados é importante no controle de processos de alguns reatores encontrados na indústria petroquímica. Esse trabalho tem como objetivo principal discutir a possibilidade da medição da concentração de partícula pela Tomographic-PIV analisando o cenário atual da técnica. O princípio de funcionamento, reconstrução tomográfica e as limitações da Tomographic-PIV são discutidos e uma breve revisão das aplicações PIV em sistemas partículados é apresentado.
\end{abstract}

\section{PIV E SISTEMAS PARTÍCULADOS}

A observação, a medição, o controle, o cálculo, a comunicação e o registro de dados, bem como a instrumentação associada, são atributos e meios fundamentais em qualquer ramo da engenharia. A técnica de medição é um conjunto de operações que têm por objetivo determinar o valor de uma grandeza. Estas operações podem ser realizadas manualmente ou automaticamente (CAMPILHO, 2000). Nos últimos 20 anos, alguns escoamentos, com uma única fase ou multifásicos, são investigados pela técnica de velocimetria por imagem de partícula (Particle Image Velocimetry - PIV) por causa da sua capacidade de visualizar e quantificar o campos de velocidade (2D ou 3D) instantânea e média dentro de uma área ou volume de investigação (Figura 1) com o objetivo de identificar estruturas e padrões de escoamento (RAFFEL et al, 2007; ADRIAN e WESTERWEEL, 2011).
Figura 1 - Particle Image Velocimetry (PIV)

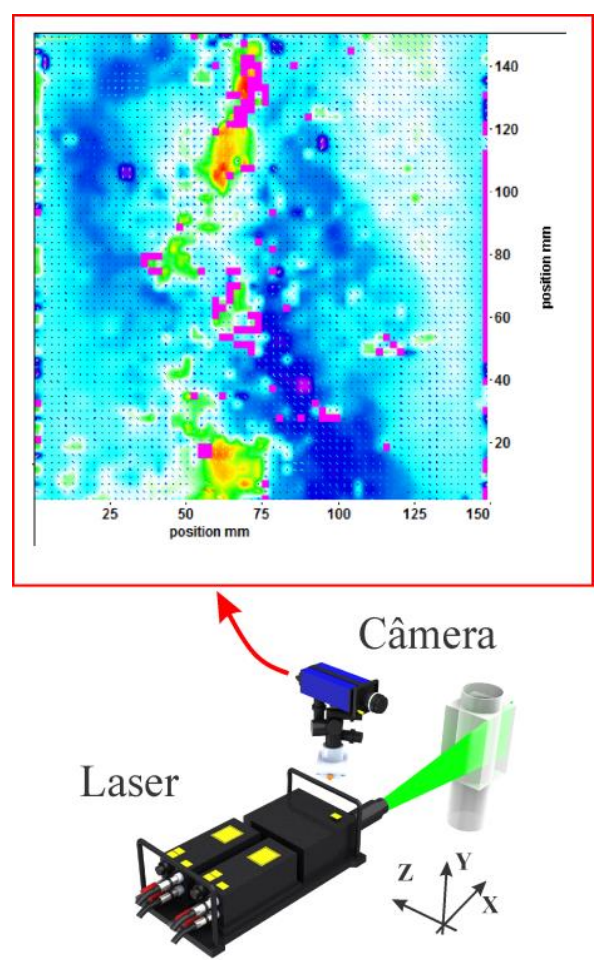

Fonte: (Adaptado de AMARAL, 2013a). 
A PIV Clássica 2D (Figura 1) emprega partículas traçadoras que seguem fielmente o escoamento do fluido, para extrair indiretamente a velocidade do fluido. As partículas no fluido são iluminadas por uma folha de luz pulsada. A fonte de luz pode ser Laser ou LED. As partículas traçadoras espalham luz em um sensor CCD ou CMOS, localizada geralmente a $90^{\circ}$ da folha de luz, de modo que o plano do objeto, em foco, coincida com a fatia iluminada do fluido. As imagens formadas no sensor são préprocessadas, processadas e pós-processadas com o objetivo de extrair as estruturas de escoamento garantindo a qualidade da medida. Em vez de determinar o deslocamento de partículas individuais como é realizado na técnica Particle Tracking Velocimetry (PTV), o algoritmo PIV correlaciona o padrão de movimento de grupos de imagens de partículas, separados em um intervalo de tempo suficientemente pequeno no ordem de $10^{-6}$ segundos, em pequenas regiões conhecidas como janelas de interrogação, fornecendo um vetor deslocamento por janela (PRASAD, 2000). O potencial da PIV Clássica 2D pode ser estendido para obter os três componentes de velocidade, no plano investigado, utilizando duas câmaras, utilizando diferentes projeções, que registram o movimento das partículas traçadoras. Esta técnica é referida como Stereoscopic-PIV (Figura 2) ou Stereo-PIV (WILLERT, 1997). A técnica TomographicPIV possibilita a determinação da velocidade em 3D utilizando geralmente 4 câmeras com projeções diferentes. A Tomographic-PIV (Figura 3) tem como objetivo representar o padrão de deslocamento das partículas traçadoras, no espaço físico, por imagens de partículas reconstruídas no espaço de imagem (ELSINGA et al., 2006a). Desde o aparecimento da Tomographic-PIV em 2005, vários grupos de pesquisas iniciaram $\mathrm{o}$ desenvolvimento da técnica em várias direções, com os primeiros esforços dedicados à reconstrução tomográfica. As aplicações concentraram-se na avaliação de estruturas instantâneas 3D de escoamentos turbulentos (SCARANO, 2013).

Figura 2 - Stereoscopic-PIV (Stereo-PIV)

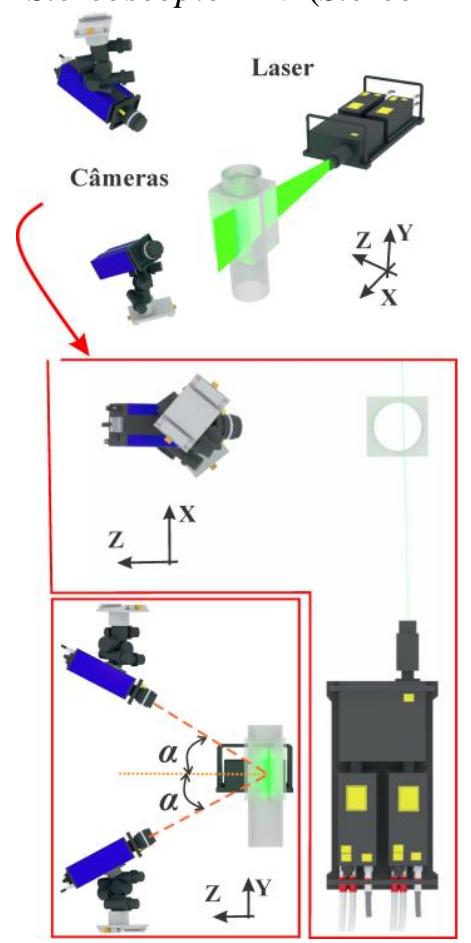

Fonte: (Adaptado de AMARAL, 2013a).

Figura 3 - Tomographic-PIV (Tomo-PIV) Distribuição 3D da Velocidade

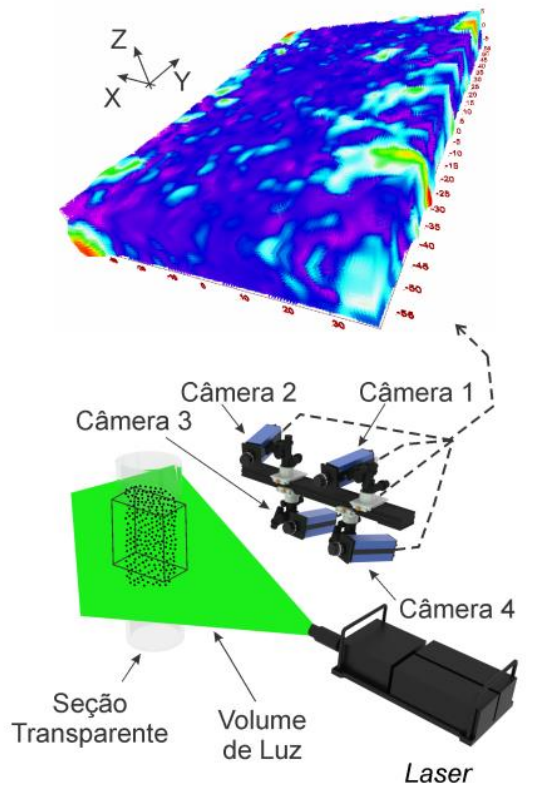

Fonte: (Adaptado de AMARAL et al, 2012a). 
Shi (2007) utilizou a PIV Clássica 2D na investigação do movimento das partículas e das propriedades dos clusters (aglomerados) em um escoamento gás-sólido em um riser, de seção quadrada, de um leito fluidizado circulante. A câmera utilizada na investigação possuia uma frequência de 500 frames por segundo. $\mathrm{O}$ autor não apresentou o campo vetorial para toda região investigada. A velocidade das partículas só foi determinada nas regiões de baixa concentração. Gryczka et al. (2008) analisaram a velocidade das partículas, em um leito de jorro com duas fendas simétricas, pela PIV Clássica 2D sem o uso da luz laser (condição não ideal para a determinação de campos de velocidade por PIV). Os autores também só conseguiram determinar a velocidade das regiões diluidas (concentração baixa de partículas) do leito analisado. A PIV não pode ser usada na investigação da velocidade em sistemas opacos (RAFFEL et al, 2007; ADRIAN e WESTERWEEL, 2011). He et al. (2009) estudou o escoamento gás-sólido turbulento em um riser, de seção retangular, de um leito fluidizado circulante utilizando a PIV Clássica 2D e análise numérica. $\mathrm{O}$ autor relata que não obteve imagem de boa qualidade, na análise feita pela PIV, com o aumento da fração volumétrica de sólidos, pois não foi possível a penetração da luz laser em zonas concentradas de partículas. A análise da velocidade na fase densa não foi discutida com detalhes pelos autores.

Amaral et al. (2012a) determinaram a velocidade em três dimensões da fase partícula, de um escoamento gás-sólido, na seção de um riser circular de um leito circulante utilizando a Tomographic-PIV. Os autores analisaram superficialmente as principais etapas (calibração, autocalibração volumétrica, pré-processamento e processamento das imagens para obtenção da distribuição da velocidade 3D pela Tomographic-PIV. Amaral et al. (2012b) apresentaram o primeiro trabalho sobre a
Tomographic-PIV no Brasil analisando o efeito da resolução espacial (resolução de interrogação) na análise da velocidade da fase partícula na seção do riser de um leito circulante. Nesses dois trabalhos, os autores não detalham o efeito da luz laser no tubo ou da distorção da imagem na análise da distribuição da velocidade 3D.

Amaral 2013 utilizou o método de autocalibração volumétrica de Wieneke (2013) para compensar a deformação nas imagens das 4 câmeras, de um sistema Tomographic-PIV, causada pela curvatura do riser de um leitocirculante. Nesse trabalho foi analisado também o arranjo das 4 câmeras CCD e o efeito do pré-processamento nas imagens obtidas. Amaral 2013b utilizou o pacote "3D Particle Density" encontrado no software Davis 8.13 desenvolvido pela LaVision que determina a concentração de partículas reconstruídas (ou número de partículas reconstruídas por volume de interrogação ou por metros cúbicos. Alguns autores chamam de $3 D$ Particle Density) para estudar o efeito da distribuição de particulados não homogênea na autocalibração volumétrica.

A determinação da distribuição de particulados na engenharia química é importante para o controle e otimização de vários processos na indústria petroquímica, por exemplo. Esse trabalho tem como objetivo discutir a possibilidade da técnica de medida Tomographic-PIV de determinar a concentração de partículados. O cenário atual de desenvolvimento da técnica também é apresentado.

\section{TOMOGRAPHIC-PIV}

$\mathrm{O}$ princípio de funcionamento da técnica Tomographic-PIV, desenvolvivo por Elsinga et al. (2005a e 2005b), é apresentado na Figura 4. 
Figura 4 - Princípio de funcionamento da Tomographic-PIV

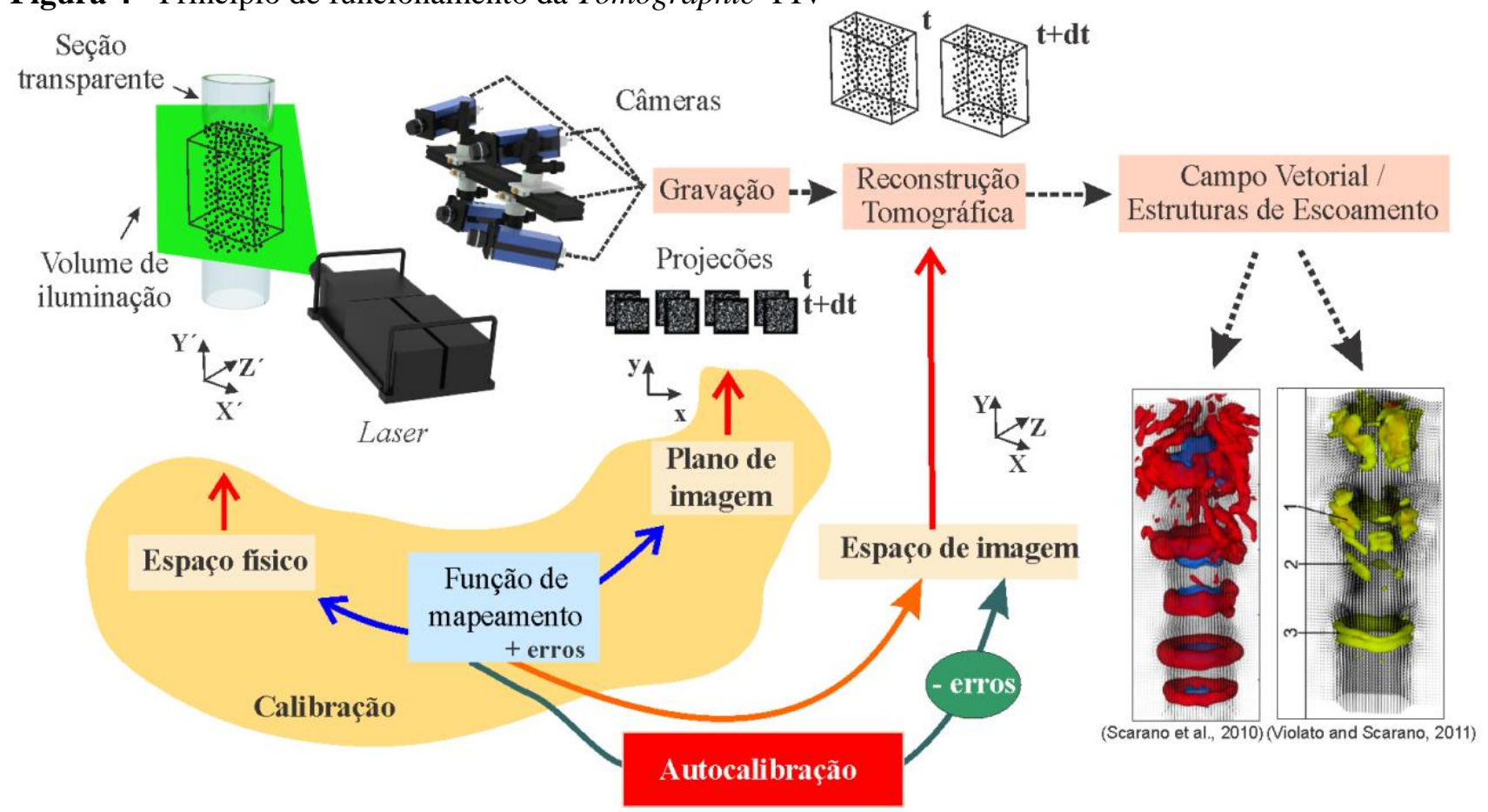

Fonte: (AMARAL, 2013a).

O padrão de luz espalhada das partículas é registrado simultaneamente por 24 câmeras CCD (ou CMOS) com projeções diferentes. A distribuição de partículas em 3D (o objeto) é reconstruída como uma distribuição de intensidade da luz em 3D a partir de suas projeções sobre as matrizes CCD (ou CMOS) por triangulação das diferentes linhas de visão. Dois volumes reconstruídos separados por um intervalo de tempo são correlacionados para determinar a distribuição de velocidade 3D (ELSINGA et al., 2006a).

Em aplicações PIV, existem três tipos de concentração de partícula: concentração de partícula $\left(\mathrm{C}_{\mathrm{p}}\right)$, concentração da imagem de partícula ( $p p p)$ e concentração da imagem de partícula reconstruída $\left(\mathrm{N}_{\mathrm{R}}\right)$. Como mostrado na Figura 5, Cp, ppp e $\mathrm{N}_{\mathrm{R}}$ são determinados no espaço físico, plano de imagem e no espaço de imagem, respectivamente.

A questão é: É possível estimar Cp por $\mathrm{N}_{\mathrm{R}}$ ? Para responder esta pergunta, é importante compreender como é feita a reconstrução tomográfica assim como o $\mathrm{N}_{\mathrm{R}}$ é calculado a partir do volume reconstruído.

Figura 5 - Três tipos de concentrações de partícula encontradas em Aplicações PIV

Espaço Físico
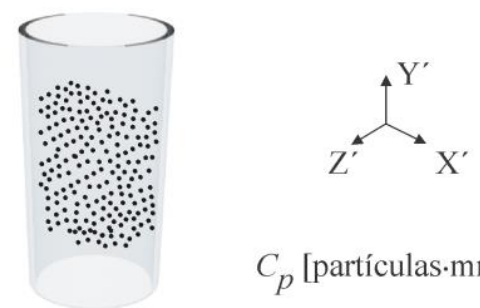

$C_{p}$ [partículas $\left.\cdot \mathrm{mm}^{-3}\right]$

Imagens 2D

ppp [imagens de partículas por pixel]
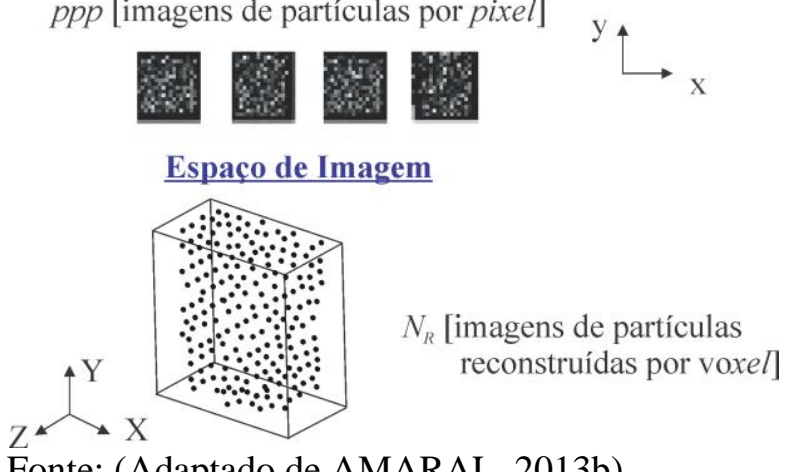

Fonte: (Adaptado de AMARAL, 2013b). 


\section{CONCENTRAÇÃO DE IMAGEM DE PARTÍCULA RECONSTRUÍDA}

A reconstrução tomográfica (Figura 6) envolve a estimativa das distribuições 3D das intensidades da imagem de partículas a partir de um número limitado de imagens em 2D (MICHAEL e YANG, 1991).

Figura 6 - Exemplo de reconstrução tomográfica de uma única partícula

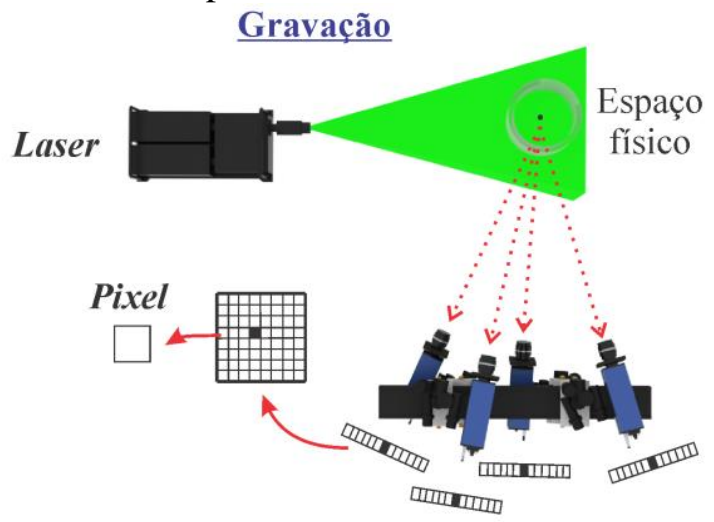

Plano de Imagem

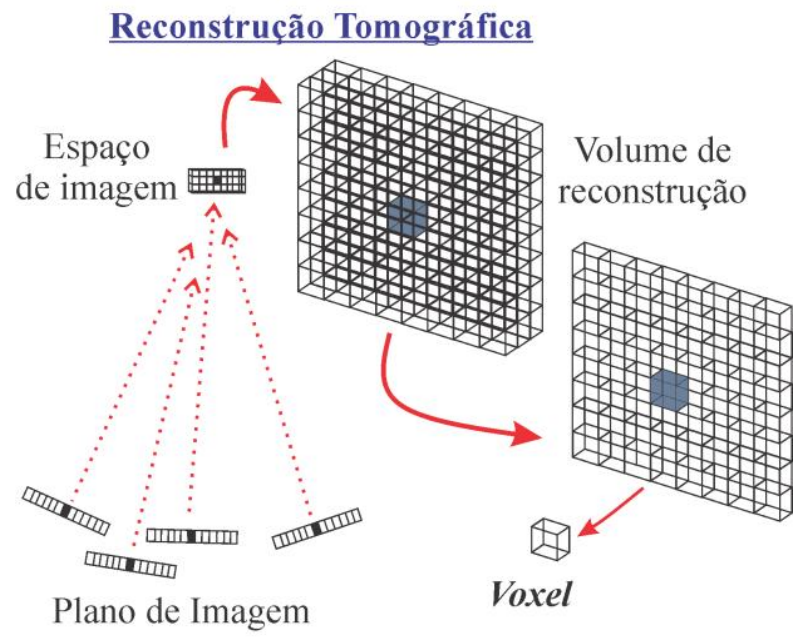

Fonte: (Adaptado de AMARAL, 2013a).

Geralmente o objeto (distribuição das partículas em 3D) é reconstruído como uma distribuição da intensidade da luz tridimensional empregando a técnica de reconstrução algébrica multiplicativa (MART) (HERMAN e LENT, 1976). O objeto é representado na forma de dados discretos, como uma matriz tridimensional de elementos de voxels (pixels ${ }^{3}$ ) cúbicos, e o conjunto de equações lineares (na hipótese de superposição dos efeitos) de modelagem do sistema de imagem são resolvidos de forma iterativa. O objeto é discretizado por uma série de voxels tridimensionais no espaço de imagem $(X, Y, Z)$, com a intensidade da luZ representada por $\mathrm{E}(\mathrm{X}, \mathrm{Y}, \mathrm{Z})$. A projeção da distribuição da intensidade da luz em cada pixel da imagem $\left(\mathrm{x}_{\mathrm{i}}, \mathrm{y}_{\mathrm{i}}\right)$ fornece a distribuição da intensidade de imagem $\mathrm{I}\left(\mathrm{x}_{\mathrm{i}}, \mathrm{y}_{\mathrm{i}}\right)$, para cada sentido da visão, que é escrita como uma equação linear (Equação 1). $\mathrm{w}_{\mathrm{i}, \mathrm{j}}$ é $\mathrm{o}$ coeficiente de ponderação que descreve a contribuição da intensidade do j-ésimo voxel $\mathrm{E}\left(\mathrm{X}_{\mathrm{j}}, \mathrm{Y}_{\mathrm{j}}, \mathrm{Z}_{\mathrm{j}}\right)$ com a intensidade de luz do iésimo pixel $\mathrm{I}\left(\mathrm{x}_{\mathrm{i}}, \mathrm{y}_{\mathrm{i}}\right) . \mathrm{N}_{\mathrm{vi}}$ é o número total de voxels na linha de visão, eixo que liga a câmera ao alvo, correspondente ao i-ésimo pixel $\left(\mathrm{x}_{\mathrm{i}}, \mathrm{y}_{\mathrm{i}}\right)$ (ELSINGA, 2008).

$$
\sum_{j \in N_{v i}} w_{i, j} E\left(X_{j}, Y_{j}, Z_{j}\right)=I\left(x_{i}, y_{i}\right)
$$

Na prática, a MART é implementada como uma técnica iterativa, com uma atualização do sistema de equações com base na relação entre a intensidade de luz no pixel medido e a projeção do objeto, juntamente com um parâmetro apropriado escalar de relaxamento. Para um valor inicial $\mathrm{E}^{0}\left(\mathrm{X}_{\mathrm{j}}, \mathrm{Y}_{\mathrm{j}}, \mathrm{Z}_{\mathrm{j}}\right)$, a atualização do objeto é feita em cada iteração completa (Equação 2). $\mu$ é o parâmetro de relaxamento escalar (HERMAN e LENT, 1976), com a propriedade de que $\mu \in$ $(0,1)$ para a técnica MART. A magnitude da atualização é, por conseguinte, determinada pela relação da intensidade de luz no pixel medido $\mathrm{I}\left(\mathrm{x}_{\mathrm{i}}, \mathrm{y}_{\mathrm{i}}\right)$ com a projeção do atual objeto $E^{k}\left(X_{j}, Y_{j}, Z_{j}\right)$. O esquema MART exige que $E\left(X_{j}, Y_{j}, Z_{j}\right)$ e $I\left(x_{i}, y_{i}\right)$ sejam definidas positivas (ELSINGA, 2008). 
$E^{k+1}=E^{k} \cdot\left[\frac{I\left(x_{i}, y_{j}\right)}{\sum_{j \in N_{v i}} w_{i, j} E^{k}}\right]^{\mu \cdot w_{i, j}}$

Um problema comum que surge na reconstrução de imagens de partículas é a presença de regiões não nulas de intensidade que não correspondem às posições de partículas reais (ELSINGA et al., 2006b). As partículas fantasma são definidas como picos de intensidade que não correspondem à localização real de partículas medidas, mas sim são criados pela presença de múltiplos locais de partícula que podem satisfazer as imagens gravadas (ATKINSON et al., 2011). A intensidade das partículas fantasma tende a ter uma intensidade mais baixa do que a das partículas (ELSINGA et al., 2006b; ATKINSON e SORIA, 2009). O número de partículas fantasma produzidas pela técnica de reconstrução MART pode ser comparável e, muitas vezes maior do que o número de partículas reais, e depende principalmente do número de pontos de vista simultâneos, da concentração de partículas e da espessura do volume iluminado (MAAS et al., 1993).

De acordo com Elsinga et al. (2006b), a proporção de partículas $\mathrm{N}_{\mathrm{p}}$ e partículas fantasma $\mathrm{N}_{\mathrm{f}}$ é dada pela Equação $3 \cdot \mathrm{N}_{\mathrm{C}}$ representa o número de câmeras, $\mathrm{A}_{\mathrm{p}}$ é a área da imagem da partícula em pixel, ppp (partículas por pixel) é a concentração de partículas na imagem e $\mathrm{L}_{z}$ é o comprimento do volume ao longo da direção de profundidade.

$\frac{N_{p}}{N_{f}}=\frac{1}{p p p^{N_{c}-1} \cdot A_{p}^{N_{c}} L_{z}}$

Simulações feitas por Elsinga et al. (2006a) demonstram o efeito da concentração de partículas reconstruídas na qualidade do volume reconstruído Q. Os autores definiram o fator de qualidade Q (Equação 4) como sendo a correlação normalizada da distribuição 3D da intensidade da imagem de partícula exata $\mathrm{E}_{0}(\mathrm{X}, \mathrm{Y}, \mathrm{Z})$ e reconstruída $\mathrm{E}_{1}(\mathrm{X}, \mathrm{Y}, \mathrm{Z})$, variando o número de câmeras utilizadas. Os resultados, Figura 7, mostram que uma alta concentração de imagens de partículas diminui a qualidade do volume reconstruído. A máxima concentração de imagem de partícula que resulta em uma qualidade aceitável do volume reconstruído é de 0,075 e 0,15 partículas por pixel ( $p p p$ ) para um sistema de três e quatro câmeras, respectivamente.

$$
Q=\frac{\sum_{X, Y, Z} E_{1}(X, Y, Z) \cdot E_{0}(X, Y, Z)}{\sqrt{\sum_{X, Y, Z} E_{1}^{2}(X, Y, Z) \cdot E_{0}^{2}(X, Y, Z)}}
$$

Figura 7 - Qualidade do volume reconstruído em função da concentração de partículas e do número de câmeras

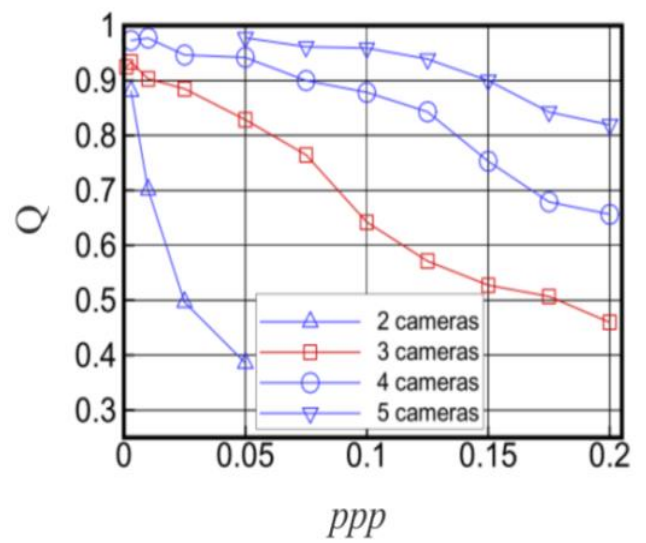

Fonte: (ELSINGA et al., 2006a)

Um parâmetro importante introduzido por Keane e Adrian (1990) é a concentração da fonte $\mathrm{N}_{\mathrm{S}}$ (source density), Equação 5, que leva em consideração a concentração da imagem de partícula no frame e o diâmetro da imagem de partícula, em que $d_{\tau}^{*}$ é definido como o diâmetro da imagem de partícula normalizado. Os autores utilizaram o Ns para estudar a qualidade do campo vetorial obtido para diferentes configurações de processamento PIV (estratégias de interrogação PIV) 


$$
N_{S}=p p p \cdot \frac{\pi \cdot d_{\tau}^{*}}{4}
$$

A relação entre a concentração da fonte $\left(\mathrm{N}_{\mathrm{S}}\right)$ e a concentração de partícula $\left(C_{p}\right)$ é dada pela Equação 6, em que $\mathrm{D}_{\mathrm{R}}$ é a resolução da imagem digital (pixel. $\mathrm{mm}^{-1}$ ). Nesse caso, a concentração de fonte governa diretamente a relação entre a concentração do meio e a precisão da reconstrução tomográfica. Simulações feitas por Novara et al. (2010) indicam que um volume reconstruído precisamente é obtido quando $\mathrm{N}_{\mathrm{S}}<0,5$.

$C_{p}=\frac{N_{S} \cdot D_{R}^{2}}{Z} \cdot \frac{4}{\pi \cdot d_{\tau}^{* 2}}$

As partículas reconstruídas pelo MART são, de fato, de alguma forma artificial, pois na maioria dos experimentos com difração óptica o tamanho de partícula real (espaço físico) é muito menor do que um voxel (WIENEKE, 2013). Atualmente, não há garantias de que o volume reconstruído represente exatamente o volume de medição (sem perdas/criação de partículas ou deformação da sua forma).

Amaral 2013 apresentou o efeito do erro de calibração, causado principalmente pela curvatura do riser, no volume reconstruído (Figura 8). Com esse erro, os volumes reconstruídos apresentaram “manchas" e não imagens de partículas.

\section{IMPORTÂNCIA DA ANÁLISE DA CONCENTRAÇÃO DE IMAGEM DE PARTÍCULA RECONSTRUÍDA.}

A resolução espacial em TomographicPIV está relacionada com a resolução espacial do sistema de gravação (magnificação de imagem), em pixels $/ \mathrm{mm}$, e a concentração de partículas reconstruídas no volume $\left(\mathrm{N}_{\mathrm{R}}\right)$. O número de partículas reconstruídas dentro do volume de interrogação necessário para realizar uma análise de correlação cruzada robusta varia entre 5 e 10, considerando-se que o fenômeno de perda de pares de partículas é insignificante para a análise do movimento em 3D (SCARANO, 2013).

Muitos trabalhos relacionam a resolução espacial apenas com o tamanho do sensor CCD (ou CMOS, dependendo da câmera utilizada), em pixel $x$ pixel $\left(\right.$ pixel $\left.^{2}\right)$. Para analisar pequenas estruturas de escoamentos e cálculos de gradientes de velocidade, por exemplo, é exigido um campo vetorial (3D3C) mais denso e consequentemente uma alta resolução espacial. Para isso, o efeito da concentração de partícula reconstruída por volume de interrogação na razão sinal-ruído (Signal-to-Noise Ratio - SNR) da correlação cruzada deve ser analisado.

Figura 8 - Iso-surfaces da concentração de imagem de partícula reconstruída por volume de interrogação (a) sem e (b) com o procedimento de autocalibração.

$\mathbf{N}_{\mathrm{RVI}}$ [Partículas/ volume de interrogação]
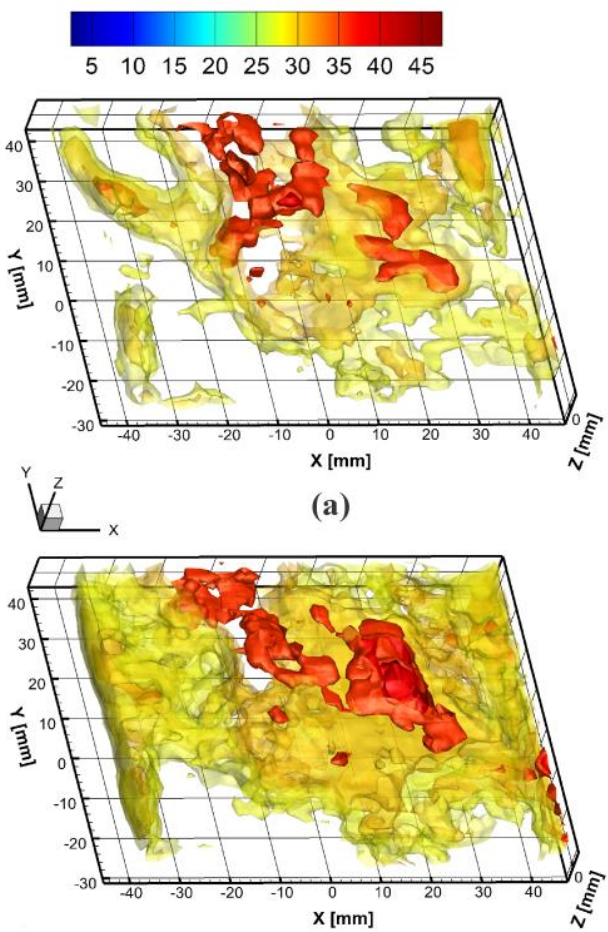

(b)

Fonte: (AMARAL, 2013b). 
Para avaliar a contribuição das partículas fantasma na medição da velocidade, não só a sua ocorrência em volumes individuais precisa ser considerado, mas também a maneira em que se adiciona ao sinal no mapa de correlação cruzada. Este é um problema de recorrência de partículas fantasma em ambos os volumes reconstruídos. Um modelo para o deslocamento da partícula fantasma, ou velocidade, é mostrado na Figura 9a (ELSINGA et al., 2011).

Figura 9 - (a) Formação de partículas fantasma que contribuam para a correlação cruzada e (b) não-correlação entre partículas fantasma

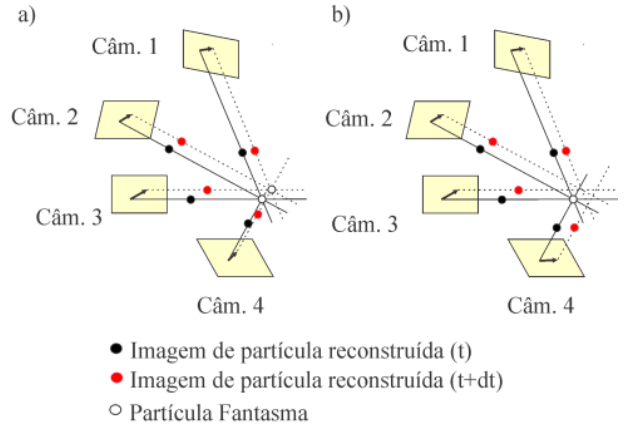

Fonte: (Adaptado de Elsinga et al. (2011) por Amaral (2013b)).

O par de partículas fantasma resultante irá afetar o mapa de correlação se, no entanto, o deslocamento de uma única destas quatro partículas (a imagem de partícula reconstruída correspondendo à câmara 4 na Figura 9b) é diferente dos outros e as linhas de visão na segunda exposição (linhas tracejadas) não mais se cruzam em um único ponto comum. Neste exemplo, uma partícula fantasma é formada a partir de quatro imagens de partículas reconstruídas de um sistema de quatro câmaras. No caso do deslocamento normal à direção de observação (entre as duas posições) ser quase igual para todas as quatro partículas (para dentro de aproximadamente um diâmetro de partícula de imagem, Figura 9a), a partícula fantasma é formada em ambas as exposições a partir das mesmas quatro imagens de partícula reconstruída e é deslocada aproximadamente pelo deslocamento médio dessas imagens de partículas reconstruídas (ELSINGA et al., 2011).

Novara et al (2010) introduziu um novo método que explora as informações obtidas em dois (ou mais ) instantes de tempo diferentes utilizando o Time-Resolved Tomographic - PIV (PIV 4D). O principal objetivo desse método (Motion Tracking Enhanced - MTE) é o de melhorar a distribuição inicial da intensidade de luz, tendo o campo de partículas nas exposições subsequentes e deformando a distribuição de partículas para o padrão equivalente à exposição anterior. $\mathrm{O}$ princípio de funcionamento é fundamentado na análise do movimento baseado na reconstrução MART e na correlação cruzada preliminar, seguido por uma deformação do objeto de acordo com o campo de velocidade. Duas versões do objeto correspondente aos mesmos instantes de tempo serão produzidas compartilhando a mesma distribuição das partículas reais (componente coerente), mas serão produzidas uma distribuição profundamente alterada das partículas fantasma (componente incoerente). As simulações numéricas mostraram que, para o mesmo número de câmeras, a qualidade da reconstrução é aumentada e os experimentos podem ser feitos com a concentração de partícula mais alta utilizando o método MART-MTE em relação aos experimentos feitos pelo MART (WIENEKE, 2013). A empresa LaVision vende um sistema Tomographic-PIV com 2 câmeras com 4M pixels que se baseia nesse método, com a restrição de concentração de imagem de partícula menor que 0,2 ppp.

\section{CONSIDERAÇÕES FINAIS}

Atualmente não é possível garantir que a concentração de partícula reconstruída no espaço de imagem pela Tomographic-PIV represente precisamente a concentração real 
de partículas traçadoras no espaço físico. Além disso, foi destacado a importância de estudar/determinar a concentração de partícula reconstruída na correlação cruzada PIV e o efeito das partículas fantasma na qualidade do volume reconstruído e da distribuição de velocidade 3D. Mesmo com essas limitações, as técnicas de medidas envolvendo DIC (Digital Image Correlation) demonstram potencial para estimar a concentração 2D de partículados em regiões diluídas. O trabalho de Warner e Smith (2014) demonstra a aplicação PIV para estimar a concentração 2D das partículas traçadoras utilizando a autocorrelação gerando uma equação empirica.

\section{REFERÊNCIAS}

ADRIAN, R. J.; WESTERWEEL, J. Particle Image Velocimetry. New York: Cambridge University Press, p. 584. 2011.

AMARAL, R. L. "Uso da Velocimetria por Imagem de Partícula (PIV) na Indústria do Petróleo", Palestra UFPE, Recife. 2013a.

AMARAL, R. L. Efeito da autocalibração volumétrica para PIV tomográfica no campo de velocidade em uma seção de um riser de um leito fluidizado circulante. Dissertação de Mestrado. FEQ-UNICAMP. Campinas-SP, 2013b.

AMARAL, R. L.; CASTILHO, G. J.; CREMASCO, M. A. Efeito da resolução de interrogação na velocimetria por imagem de partícula do tipo tomográfica na investigação do campo de velocidade da fase particulada na seção de um riser. Congresso Brasileiro de Engenharia Química (COBEQ 2012). Búzios - RJ. 2012b

AMARAL, R. L.; CASTILHO, G. J.; CREMASCO, M. A. Experimental Investigation of 3D Velocity by Tomographic
Particle Image Velocimetry (Tomo-PIV) in a Short Riser Section. Procedia Engineering, v. 42, p. 748-754, jan. 2012a.

ATKINSON, C.; COUDERT, S.; FOUCAUT, $\mathrm{J}$. The accuracy of tomographic particle image velocimetry for measurements of a turbulent boundary layer. Experiments in Fluids, v. 50, p. 1031-1056, 2011.

ATKINSON, C.; SORIA, J. An efficient simultaneous reconstruction technique for tomographic particle image velocimetry. Experiments in Fluids, v. 47, n. 4-5, p. 553568, 2009.

CAMPILHO, A. Instrumentação Eletrônica. Métodos e Técnicas de Medição. Marca-Artes Gráficas, Porto, 2010.

ELSINGA, G. E.; SCARANO, F.; WIENEKE, B.; VAN OUDHEUSDEN Tomographic particle image velocimetry. Experiments in Fluids, v. 41, n. 6, p. 933947, 11 out. 2006a.

ELSINGA, G. E.; SCARANO, F.; WIENEKE, B.; VAN OUDHEUSDEN. Assessment of Tomo-PIV for threedimensional flows. 6th International Symposium on Particle Image Velocimetry. California. 2005b

ELSINGA, G. E.; SCARANO, F.; WIENEKE, B.; VAN OUDHEUSDEN. Tomographic particle image velocimetry. 6th International Symposium on Particle Image Velocimetry. California. 2005a

ELSINGA, G. Tomographic particle image velocimetry and its application to turbulent boundary layers. Thesis. University of Technology Delft, 2008.

ELSINGA, G.; WESTERWEEL, J.; SCARANO, F.; NOVARA, M. On the 
velocity of ghost particles and the bias errors in Tomographic-PIV. Experiments in fluids, v. 50, p. 825-838, 2011.

GRYCZKA, O. HEINRICH, S.; MITEVA, V.; DEEN, N.G.; KUIPERS, J.A.M.; JACOB, M.; MÖRL, L. Characterization of the pneumatic behavior of a novel spouted bed apparatus with two adjustable gas inlets. Chemical Engineering Science, v. 63, n. 3, p. 791-814, 2008.

HE, Y. DEEN, N. G.; ANNALAND, M. VAN SINT; KUIPERS, J. A. M. Gas-Solid Turbulent Flow in a Circulating Fluidized Bed Riser: Experimental and Numerical Study of Monodisperse Particle Systems. Industrial \& Engineering Chemistry Research, v. 48, n. 17, p. 8091-8097, 2009.

HERMAN, G.; LENT, A. Iterative reconstruction algorithms. Computers in biology and medicine, v. 6, n. 3, p. 273-294, 1976.

KEANE, R.; ADRIAN, R. Optimization of particle image velocimeters. I. Double pulsed systems. Measurement Science and Technology, v. 1, p. 1202-1215, 1990.

MAAS, H.; GRUEN, A.; PAPANTONIOU, D. Particle tracking velocimetry in threedimensional flows. Experiments in Fluids, v. 15, p. 133-164, 1993.

MICHAEL, Y.; YANG, K. Recent developments in axial tomography for heat transfer and fluid flow studies. Experimental thermal and fluid science, v. 4, p. 637-647, 1991.

NOVARA, M.; BATENBURG, K. J.; SCARANO, F. Motion tracking-enhanced MART for Tomographic-PIV. Measurement science and technology, v. 21, 2010.
PRASAD, A. Particle image velocimetry Review article. Current Science, v. 79, p. 51-60, 2000.

RAFFEL, M.; WILLERT, C.; KOMPENHANS, J. Particle image velocimetry: a practical guide. Second Edi ed. Berlin Heidelberg: Springer Verlag, 2007.

SCARANO, F. Tomographic PIV: principles and practice. Measurement Science and Technology, v. 24, n. 1, p. 012001, 2013.

SCARANO, F.; BRYON, K.; VIOLATO, D. Time-resolved analysis of circular and chevron jets transition by tomo-PIV 15th Int. Symp. on Applications of Laser Techniques to Fluid Mechanics (Lisbon, Portugal), 2010

SHI, H. Experimental research of flow structure in a gas-solid circulating fluidized bed riser by PIV. Journal of Hydrodynamics, Ser. B, v. 19, n. 6, p. 712719, 2007.

VIOLATO, D. e SCARANO, F. Threedimensional evolution of flow structures in transitional circular and chevron jets. Physics Of Fluids, 23. 2011

WARNER, S. O. e SMITH, B. L. Autocorrelation-based estimate of particle image density for diffraction limited particle images. Measurement science and technology. v. 252014.

WIENEKE, B. Iterative reconstruction of volumetric particle distribution. Measurement Science and Technology, v. 24, n. 1, p. 024008, 2013.

WILLERT, C. Stereoscopic digital particle image velocimetry for application in wind tunnel flows. Measurement science and technology, v. 8, p. 1465-1479, 1997. 